\title{
Nueva metodología para la delimitación de las zonas a reparar de un hormigón afectado por corrosión
}

\section{New Methodology for the Delimitation of Areas to be Repaired of a Concrete Affected by Corrosion}

\author{
Servando Chinchón Payá, , a Javier Sánchez Montero ${ }^{\mathrm{b}}$, Luis $\mathrm{M}^{\mathrm{a}}$ Ortega Basagoitic, \\ Jesús García Mateos ${ }^{\mathrm{d}}$, Nuria Rebolledo Ramos ${ }^{\mathrm{e}}$, Julio Emilio Torres Martín \\ Jaime Peribáñez Sebastián" \\ ${ }^{a}$ PhD Chemist. Instituto Eduardo Torroja Ciencias de la Construcción (IETcc-CSIC) \\ ${ }^{b}$ PhD Eng. Chemist. Instituto Eduardo Torroja Ciencias de la Construcción (IETcc-CSIC) \\ ${ }^{c}$ Civil Eng. Retineo Ingeniería \\ ${ }^{d}$ Tech. Civil Eng. Retineo Ingeniería \\ ${ }^{e}$ Chemical Engineer. Instituto Eduardo Torroja Ciencias de la Construcción (IETcc-CSIC) \\ ${ }^{f}$ PhD Eng. Material. Instituto Eduardo Torroja Ciencias de la Construcción (IETcc-CSIC) \\ ${ }^{g}$ Civil Eng. Retineo Ingeniería
}

Recibido el 7 de junio de 2019; aceptado el 4 de abril de 2020

\section{RESUMEN}

Este artículo describe el uso en obra de un equipo portátil de fluorescencia de rayos X (FRX) para la cuantificación en obra de cloruros en muestras de hormigón extraídas de una estructura afectada por corrosión. Su aplicación permite delimitar con un criterio más adecuado las zonas a reparar, extendiendo la intervención a zonas adyacentes a las claramente dañadas, aparentemente sanas, pero en las que la concentración de cloruros en el hormigón supera el límite propuesto por la EHE.

(C)2022 Hormigón y Acero, la revista de la Asociación Española de Ingeniería Estructural (ACHE). Publicado por Cinter Divulgación Técnica S.L. Este es un artículo de acceso abierto distribuido bajo los términos de la licencia de uso Creative Commons (CC BY-NC-ND 4.0)

KEYWORDS: Reparación, refuerzo de estructuras, pFRX, cloruros.

\section{ABSTRACT}

This paper describes the use on site of a portable X-ray fluorescence equipment (pFRX) for the quantification of chlorides in concrete samples taken from a structure affected by corrosion. Its application allows to delimit with more appropriate criteria the areas to be repaired by extending the intervention to areas adjacent to those clearly damaged, apparently healthy, but in which the concentration of chlorides in the concrete exceeds the threshold proposed by the EHE.

C2022 Hormigón y Acero, the journal of the Spanish Association of Structural Engineering (ACHE). Published by Cinter Divulgación Técnica S.L. This is an open-access article distributed under the terms of the Creative Commons (CC BY-NC-ND 4.0) License

PALABRAS CLAVE: Concrete repair, structure reinforcement, pFRX, chlorides.

1 .

\section{INTRODUCCIÓN}

\subsection{Problemática de cloruros y corrosión de armaduras}

La corrosión de las armaduras, principalmente por el efecto de

* Persona de contacto / Corresponding author:

Correo-e / e-mail: servando@ietcc.csic.es (Servando Chinchón Payá). los iones cloruro, es la principal causa de disminución de la vida útil de las estructuras de hormigón armado [1-3]. Como es sabido, el hormigón es un material con un $\mathrm{pH}$ alto (básico) lo cual sitúa al acero en unas condiciones de pasividad, puesto que proporciona la formación de una capa pasiva en la superficie de la armadura [4]. Sin embargo, al tratarse de un material poroso, 
la acción del $\mathrm{CO}_{2}$ y/o los iones cloruro provoca la pérdida de esta capa de protección pasiva del acero. Mientras el $\mathrm{CO}_{2}$ produce una disminución en el $\mathrm{pH}$ del agua presente en los poros del hormigón, lo cual induce la corrosión del acero y provoca una corrosión generalizada, en el caso de los cloruros provocan la rotura de la capa pasiva de forma localizada y da lugar a una corrosión por picaduras $[5,6]$. En función del grado de deterioro o avance de la corrosión, se producen los siguientes efectos en la estructura que van disminuyendo de forma gradual su capacidad resistente y, por lo tanto, su seguridad hasta poder alcanzar el colapso parcial o total de la misma: i) pérdida de sección de la armadura, ii) pérdida de adherencia con el hormigón, iii) fisuración o incluso pérdida del recubrimiento de hormigón, y iv) fragilización o rotura de las armaduras.

Respecto a la corrosión provocada por los iones cloruros, es necesario alcanzar una concentración mínima de los mismos a la altura de la armadura para que se desencadene el proceso de corrosión. No existe una unanimidad en la literatura sobre este umbral, ni tan siquiera sobre el propio método de ensayo [2, 7] para cuantificarlos.

\subsection{Cuantificación de cloruros}

El método normalizado de referencia para la cuantificación de iones cloruros en una muestra (Método de Volhard) se basa en la baja solubilidad que tiene el cloruro de plata, compuesto que se forma cuando en una misma disolución coexisten iones cloruro e iones plata.

$$
\begin{aligned}
& \mathrm{Cl}+\mathrm{Ag}^{+} \rightarrow \mathrm{AgCl} \\
& \text { solubilidad } \mathrm{AgCl}=1.9 \mathrm{mg} / 1 \mathrm{H}_{2} \mathrm{O}
\end{aligned}
$$

Dado que la reacción anterior se produce en medio acuoso, previamente la muestra sólida que contiene los cloruros ha de digerirse, acidificándose, para que dichos iones pasen a la fase acuosa.

Todos estos procesos de preparación de la muestra, disolución y precipitación son costosos en tiempo y forma pues han de llevarse a cabo en un laboratorio habilitado y con personal específicamente formado [8].

Existen otras técnicas para la cuantificación de cloruros entre las que podemos destacar la cromatografía iónica (CI) y la fluorescencia de rayos X (FRX). Mientras que la primera de estas técnicas necesita la muestra en fase líquida, la segunda se utiliza en muestras sólidas, generalmente en polvo muy fino. Sin embargo, tanto la CI como la FRX son técnicas que necesitan dispositivos muy caros y por tanto la cuantificación de cloruros es mucho más costosa que empleando el método de Volhard. Y, en cualquier caso, hasta ahora todos esos ensayos tenían que ser realizados en laboratorio, con el consiguiente coste y pérdida de la agilidad necesaria para la toma de decisiones cuando se trata de delimitar la zona a reparar de un elemento ya dañado por corrosión de armaduras.

Los equipos de FRX portátiles se han utilizado en diferentes campos tales como el análisis de suelos en geoquímica [9-12] o para conocer la composición de aleaciones metálicas en aceros o la industria de la reutilización y reciclado [13], en el análisis de obras artísticas, restos arqueológicos y cerámicos $[14,15]$ y en estudios variados de índole medioambiental y gestión de residuos [16-19]. Sin embargo, hay muy pocos trabajos que utilicen la técnica de FRX en materiales de construcción [20] y no se han encontrado en la bibliografía científica artículos que muestren el uso de estos equipos portátiles en ese campo, y menos aún como apoyo a la identificación de patologías en hormigones.

\subsection{Actuaciones en las reparaciones por parcheo}

Una solución habitual de reparación de los daños provocados por la corrosión de armaduras (fisuras en el hormigón siguiendo la dirección de las armaduras, desprendimientos del recubrimiento de hormigón, pérdida de sección de las armaduras por la corrosión de las mismas..., etc.) cuando estos no han llegado a afectar de forma significativa a la capacidad resistente del elemento, es la conocida coloquialmente como "parcheo". Su finalidad es, primordialmente, restituir la capacidad protectora del hormigón deteriorado hacia las armaduras que recubre y evitar el riesgo asociado de desprendimiento de fragmentos del recubrimiento de hormigón que pueden dar lugar a accidentes más o menos graves en el caso de presencia o circulación de vehículos o personas por debajo de la estructura dañada .

El parcheo supone la eliminación del hormigón deteriorado, la limpieza de las armaduras corroídas y su posterior protección contra una futura corrosión mediante inhibidores o pasivantes y la sustitución del hormigón deteriorado y carbonatado o contaminado por cloruros, con un material que suele ser habitualmente un mortero de reparación.

En el coste de esta reparación influye de manera fundamental la extensión a la que deba afectar la misma, tanto en superficie como en profundidad, puesto que se traduce en volumen de hormigón original a eliminar que posteriormente debe ser sustituido por el mortero de reparación.

Esta técnica de reparación está ampliamente extendida, aunque ha presentado tradicionalmente determinados problemas que han venido siendo estudiados por los investigadores, dando lugar a numerosas referencias sobre el tema [21-24]. Estos problemas se relacionan con la aparición de fenómenos de corrosión en zonas adyacentes a las reparadas en plazos relativamente cortos después de efectuada la reparación (entre unos cuantos meses y un año [23]).

Se puede afirmar que el procedimiento de reparación descrito, que está siendo aplicado en numerosas estructuras con daños por corrosión de armaduras, no garantiza que no se produzcan nuevos daños por procesos patológicos asociados a la corrosión de las armaduras en zonas próximas a las parcheadas, si no se asegura que el hormigón es eliminado en todas las zonas en las que el frente de carbonatación o la profundidad del contenido crítico de cloruros haya alcanzado el nivel de las armaduras [21].

Si tales condiciones no se cumplen, la durabilidad de una reparación de parcheo puede resultar muy limitada en el tiempo y empezar a detectarse corrosiones en las zonas adyacentes en el plazo de unos pocos meses, que podrían incluso provocar el propio desprendimiento de la zona parcheada [21].

\subsection{Métodos actuales más utilizados en patología de hormi- gón armado para detectar corrosión en armaduras}

Para detectar posibles procesos de corrosión en armaduras se pueden utilizar dispositivos de medida de parámetros elec- 
troquímicos que puedan dar información del estado del acero del hormigón armado. Corrosímetros del estilo GECOR [25] permiten conocer, en condiciones de análisis específicas, la presencia de procesos activos de corrosión de las armaduras de refuerzo antes de que lleguen a aparecer síntomas visibles claros de la existencia de tales procesos de corrosión.

Complementariamente, las técnicas de ensayo aplicables para determinar el contenido en cloruros del hormigón o el avance del frente de carbonatación (véase el apartado 1.2 anterior) resultarán de gran utilidad para determinar si tales procesos de corrosión son debidos a uno $\mathrm{u}$ otro agente agresivo y, como se ha comentado, para fijar con un criterio lógico, la extensión de las reparaciones por parcheo.

\subsection{Objetivos de este estudio}

El objetivo de este trabajo es mostrar que el uso de un equipo de pFRX permite conocer in situ la concentración de cloruros en los puntos deseados de una estructura, sin necesidad de retrasar las intervenciones por esperar resultados de análisis en laboratorio. De esta forma se posibilita delimitar rápidamente y con más objetividad las áreas a reparar.

\section{2}

\section{APLICACIÓN EN OBRA. METODOLOGÍA.}

Se expone la aplicación llevada a cabo en elementos de un puente que presentaba daños en el hormigón por corrosión de armaduras, razón por la que se había planteado su reparación. En la realización del trabajo se han empleado un corrosímetro GECOR y un equipo portátil de FRX.

\subsection{Medidas in situ de parámetros de corrosión}

La obtención de parámetros electroquímicos se realizó in situ con el equipo GECOR que permite medir potencial de corrosión, resistividad y velocidad de corrosión [25]. Para realizar estas medidas únicamente es necesario tener una conexión eléctrica con la armadura en un punto, posteriormente las medidas se realizan de forma no destructiva a través del recubrimiento del hormigón. El equipo permite hacer un mapeo del potencial de corrosión y la resistividad que permite identificar las zonas que se encuentran activas y las que se encuentran pasivas. Posteriormente, se realizan las medidas de intensidad de corrosión (en $\mu \mathrm{A} / \mathrm{cm}^{2}$ ) mediante el método confinado de la corriente, a través de las cuales se puede estimar la velocidad instantánea de pérdida de sección en la armadura (en mm/año [25-28].

La interpretación de las medidas de potencial de corrosión se suele hacer calificando el riesgo según el criterio (Norma UNE 112083 [29]) que se recoge en la Tabla 1.

TABLA 1.

Clasificación en niveles del potencial de corrosión

\begin{tabular}{cl}
\hline Ecorr vs. Cu/CuSO4 $(\mathrm{mV})$ & Probabilidad de corrosión \\
$>-275$ & $10 \%$ \\
-275 a -425 & $50 \%$ \\
$<-425$ & $90 \%$
\end{tabular}

En relación con la resistividad del hormigón, valores de esta superiores a 2000 S.m no permiten distinguir entre acero en estado activo o pasivo de corrosión. Las velocidades de corrosión serán bajas, independientemente del contenido de cloruros o nivel de carbonatación. Es, por tanto, un parámetro importante a la hora de interpretar las medidas de intensidad de corrosión puesto que intensidades de corrosión nulas o muy bajas con resistividades altas no implican que no puedan transformarse en intensidades altas cuando el hormigón esté húmedo y la resistividad, por tanto, baje.

\subsection{Equipo portátil de FRX}

El equipo portátil de FRX utilizado es un equipo Olympus Innov-X, modelo Delta. Se dispone también de Workstation para conectar el equipo a un ordenador y monitorizar los ensayos desde el mismo.

Para la óptima cuantificación de cloruros en muestras de hormigón, se realizó en laboratorio una calibración previa comprobando la respuesta instrumental resultando necesario aplicar un factor de corrección de 1.17 a los valores obtenidos con el equipo de pFRX.

Ya en la obra, el equipo se conectó a un ordenador portátil para el manejo del software, y ambos a un generador externo para aportarles la necesaria energía.

La extracción de las muestras se realizó mediante taladro con broca especial para hormigón. Se utilizó un recipiente de plástico que, a modo de embudo, recogiera todo el polvo generado durante el taladro minimizando al máximo las pérdidas. El polvo fue trasvasado a unos recipientes cilíndricos que a modo de portamuestras se introdujeron en la Workstation del equipo de pFRX para el análisis.

\subsection{Datos de la obra}

Se trata de un viaducto con vanos isostáticos de luces variables en torno a los 13 a $15 \mathrm{~m}$. Las pilas, de altura variable, tienen un doble fuste circular, sobre los que se empotra un dintel tipo martillo, de canto constante en la zona central entre fustes y variable en los extremos.

El elemento A corresponde a un fuste del viaducto contaminado por cloruros debidos al uso de sales de deshielo (ver figura 1). El elemento no presenta problemas de fisuración, ni daños visuales y, por tanto, no es considerado como un elemento a reparar dentro de la estructura.

La figura 2 muestra una fotografía del segundo elemento estudiado.

En este caso, el elemento B analizado presenta zonas reparadas (puntos A y B) y zonas no reparadas (puntos 1-15).

Las camas o asientos de apoyos, identificados como elemento C (figura 3) estaban aparentemente más degradados y eran de un hormigón más pobre.

\subsection{Análisis}

Se realizaron varios análisis en diferentes puntos de un fuste y del dintel ilustrados en las fotografías de las imágenes figura 1 y figura 2. Los puntos de dichas figuras ubican las zonas analizadas. En ambos elementos se obtuvieron (1) valores electroquímicos mediante GECOR, primero un mapeo de probabilidad 




Figura 1. Fuste con puntos donde se llevaron a cabo los análisis. Elemento A.

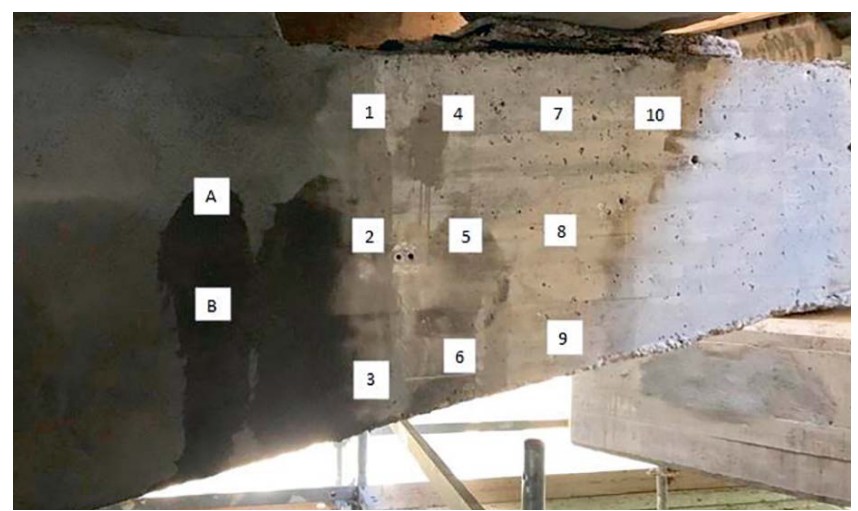

Figura 2. Dintel con puntos donde se llevaron a cabo los análisis. Elemento B.
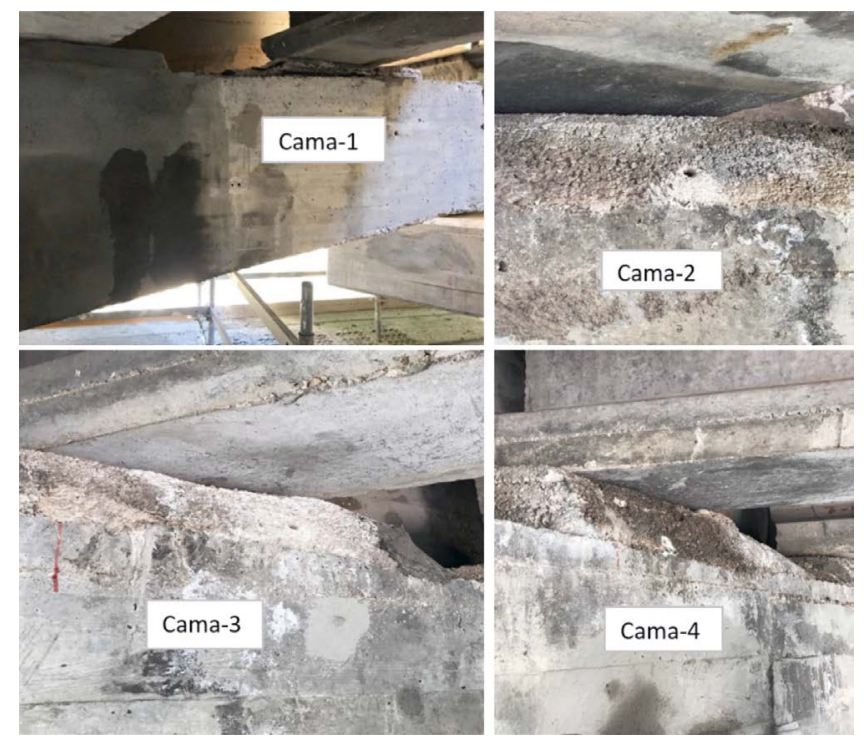

Figura 3. Camas de asiento. Elemento C. de corrosión y después de velocidad de corrosión cuando fuera necesario y (2) se cuantificó la concentración de cloruros mediante pFRX aunque no de todos los puntos señalados.

La figura 3 ilustra también cuatro zonas, de cuatro camas de asiento, también analizadas, aunque esta vez solo se cuantificó la cantidad de cloruros presente en el hormigón.

Dado que la EHE [30] establece un umbral de cloruros de $0,6 \%$ en relación al peso de cemento para considerar que la concentración de cloruros es lo suficientemente importante para provocar daños por corrosión, se hace necesario suponer una dosificación de una cierta cantidad de kg de cemento por m3 de hormigón, para poder así transformar los datos de cuantificación de cloruros aportados por el equipo a las mismas unidades de porcentaje en peso de cemento y comprobar si la cantidad de cloruros supera el límite de la norma antes indicado.

\section{3.}

\section{RESULTADOS Y DISCUSIÓN}

Se muestran a continuación los principales resultados obtenidos en todas las muestras/zonas analizadas.

\section{Elemento A.}

Se realizan medidas electroquímicas in situ con el equipo GECOR. La tabla 2 recoge los datos de potencial de corrosión y resistividad del hormigón. Los potenciales de corrosión obtenidos sitúan la estructura en un riesgo de corrosión en torno al $50 \%$, sin embargo, las medidas de resistividad son muy superiores a $2000 \Omega \cdot \mathrm{m}$, lo que indica que la estructura está muy seca y el proceso de corrosión está inactivo. Se intentaron llevar a cabo medidas cuantitativas de intensidad de corrosión, pero debido a la alta resistividad obtenida no fue posible.

TABLA 2.

Mapeo de probabilidad de corrosión. Datos obtenidos mediante GECOR en el elemento de estudio $\mathrm{A}$.

\begin{tabular}{ccc}
\hline Elemento A & $\begin{array}{c}\text { Ecorr vs. } \mathrm{Cu} / \mathrm{CuSO} 4 \\
(\mathrm{mV})\end{array}$ & $\begin{array}{c}\text { Resistividad } \\
\left(\Omega^{1} \mathbf{m}\right)\end{array}$ \\
\hline $\mathbf{1}$ & -446 & 7560 \\
$\mathbf{2}$ & -394 & 9150 \\
3 & -355 & 9990 \\
4 & -431 & 9550 \\
\hline
\end{tabular}

Los datos de potencial de corrosión indican un posible problema futuro de corrosión que en el momento puntual en el que se realizaron las medidas podría estar inactivo, pero que, tras un periodo de lluvias o época de invierno, podría volver a activarse, degradando un elemento que no ha sido contemplado durante los procesos de reparación.

La tabla 3 recoge la concentración de cloruros en las muestras analizadas in situ, que corresponden a los puntos 2 y 3 . Los resultados se expresan en $\%$ de cloruros respecto a peso de hormigón (p.h) y \% de cloruros respecto a peso de cemento (p.c.) en dos hipótesis (suponiendo un contenido de 275 (p.c. 1) y 400 (p.c.2) $\mathrm{kg}$ de cemento por $\mathrm{m}^{3} \mathrm{de}$ hormigón). 
TABLA 3.

Resultados de cuantificación de cloruros en muestras extraídas del elemento A, donde $\mathrm{D}$ es la profundidad de extracción de muestra

\begin{tabular}{|c|c|c|c|c|c|c|}
\hline \multirow{2}{*}{$\begin{array}{l}\mathrm{D} \\
\mathrm{en} \\
\mathrm{cm}\end{array}$} & \multicolumn{3}{|c|}{ Punto 2} & \multicolumn{3}{|c|}{ Punto 3} \\
\hline & \%p.h & $\begin{array}{c}\% \text { p.c. } \\
1\end{array}$ & $\begin{array}{c}\% \text { p.c. } \\
2\end{array}$ & \%p.h & $\begin{array}{c}\text { \%p.c. } \\
1\end{array}$ & $\begin{array}{c}\text { \%p.c. } \\
2\end{array}$ \\
\hline 0 & 0,092 & 0,771 & 0,530 & 0,077 & 0,644 & 0,443 \\
\hline 1 & 0,087 & 0,728 & 0,500 & 0,084 & 0,703 & 0,483 \\
\hline 2 & 0,084 & 0,705 & 0,485 & - & - & - \\
\hline 3 & 0,067 & 0,563 & 0,387 & - & - & - \\
\hline $4^{*}$ & 0,056 & 0,465 & 0,320 & - & - & - \\
\hline
\end{tabular}

*hormigón en contacto directo con la armadura

Los porcentajes de cloruros obtenidos en las muestras extraídas en el punto 2 en contacto con la armadura ( $4 \mathrm{~cm}$ de distancia) se acercan en zonas próximas a la armadura al valor del $0,6 \%$ en peso de cemento para la comprobación del Estado Límite en relación con la corrosión de las armaduras pasivas para la hipótesis de dosificación de cemento baja. La misma tendencia se observa en el punto 3 , donde los datos en superficie y a 1 $\mathrm{cm}$ de profundidad arrojan valores similares. Por otro lado, si se supone una dosificación más alta de cemento por m3 de hormigón $(400 \mathrm{~kg} / \mathrm{m} 3)$, todos los valores resultarían inferiores a $0,55 \%$. Es decir, se acercan al umbral límite de la EHE, pero no lo alcanzan. Dado que este umbral debe haberse adoptado con un criterio conservador, eso explicaría la ausencia de daños por corrosión en este momento en el elemento.

Sin embargo, nos encontramos ante una estructura contaminada por cloruros, que de no ser tratada podría presentar deterioro en un plazo breve.

\section{Elemento $B$}

En la tabla 4 se muestran las medidas de potencial de corrosión, resistividad e intensidad de corrosión. Las medidas se han realizado en dos fechas diferentes, con el fin de estudiar cómo evolucionaban los parámetros electroquímicos en la zona reparada.

La acotación de la zona de reparación (delimitación de la reparación por parcheo), se ha realizado mediante inspección visual y sónica.

Los puntos 1-15 se ubican en zonas no reparadas mientras que los puntos A y B corresponden a dos puntos de la zona ya reparada.

Los parámetros electroquímicos de corrosión muestran una estructura donde la zona no reparada (puntos del 1 al 15) presenta potenciales de corrosión más positivos de $-275 \mathrm{mV}$, con resistividades que se sitúan en un amplio rango situado entre $512 \Omega \cdot \mathrm{m}$ y más de $5000 \Omega \cdot \mathrm{m}$, lo que permite verificar que, en aquellos puntos con resistividades bajas no existe, actualmente, un proceso de corrosión.

Los puntos A y B muestran potenciales de corrosión que se sitúan en la zona de probabilidad del 50\%, resistividades del hormigón bajas, donde esta variable deja de ser el parámetro controlante y velocidades de corrosión muy por encima de $0,1 \mu \mathrm{A} / \mathrm{cm}^{2}$ (límite inferior considerado para el umbral de despasivación del acero), situándose en niveles altos de corrosión. Estos valores, obtenidos a las 24 horas de la reparación, pueden llevar a errores de interpretación, ya que no son atribuibles a una incorrecta reparación, sino a que no se había producido el proceso de repasivación de los aceros tras la limpieza y parcheo realizados.

Dos semanas después de la reparación, los valores de velocidad de corrosión disminuyeron significativamente, si-

TABLA 3.

Potencial de corrosión (vs. Cu/CuSO4), resistividad e intensidad de corrosión. Datos obtenidos mediante GECOR en el elemento de estudio B.

\begin{tabular}{|c|c|c|c|c|c|c|}
\hline \multirow[b]{2}{*}{ Elemento B } & \multicolumn{3}{|c|}{24 horas tras la reparación } & \multicolumn{3}{|c|}{15 días tras la reparación } \\
\hline & $\begin{array}{c}\text { Ecorr vs. } \\
\mathrm{Cu} / \mathrm{CuSO}_{4} \\
(\mathrm{mV})\end{array}$ & $\begin{array}{c}\text { Icorr } \\
\left(\mu \mathrm{A} / \mathrm{cm}^{2}\right)\end{array}$ & $\begin{array}{c}\text { Resistividad } \\
\left(\Omega^{1} \mathrm{~m}\right)\end{array}$ & $\begin{array}{c}\text { Ecorr vs. } \\
\mathrm{Cu} / \mathrm{CuSO}_{4} \\
(\mathrm{mV})\end{array}$ & $\begin{array}{c}\text { Icorr } \\
\left(\mu \mathrm{A} / \mathrm{cm}^{2}\right)\end{array}$ & $\begin{array}{c}\text { Resistividad } \\
\qquad\left(\Omega^{1} \mathrm{~m}\right)\end{array}$ \\
\hline 1 & -260 & - & 512 & - & - & - \\
\hline 2 & -278 & 0,086 & 2320 & -270 & 0,080 & 2750 \\
\hline 3 & -272 & 0,053 & 2700 & - & - & - \\
\hline 4 & -260 & - & 513 & - & - & - \\
\hline 5 & -254 & - & 650 & -245 & 0,060 & 3960 \\
\hline 6 & -183 & - & 3580 & - & - & - \\
\hline 7 & -265 & - & 2000 & - & - & - \\
\hline 8 & -221 & 0,090 & 2560 & -256 & 0,120 & 1052 \\
\hline 9 & -118 & - & 5370 & - & - & - \\
\hline 10 & -220 & - & 1960 & - & - & - \\
\hline 11 & -195 & - & 8110 & - & - & - \\
\hline 12 & -101 & - & 9060 & - & - & - \\
\hline 13 & -126 & - & 8660 & - & - & - \\
\hline 14 & -95 & - & 9990 & - & - & - \\
\hline 15 & -86 & - & 9990 & - & - & - \\
\hline A & -312 & 2,906 & 98 & -225 & 0,450 & 50 \\
\hline B & -292 & 1,056 & 400 & -282 & 0,160 & 456 \\
\hline
\end{tabular}


tuándose en valores de corrosión bajos $\left(0,1-0,5 \mu \mathrm{A} / \mathrm{cm}^{2}\right)$, 1o que hace suponer que se estaba ya formando la capa pasiva. Para poder comprobar la eficacia total de la reparación sería conveniente efectuar un seguimiento de la misma con más tiempo, pues es esperable que a los 30 días la velocidad de corrosión baje hasta los valores inferiores a $0,1 \mu \mathrm{A} / \mathrm{cm}^{2}$.

En la tabla 5 se puede observar la concentración de cloruros en las muestras analizadas en esta zona, correspondientes a los puntos 2 y 11 , siendo el 2 el más cercano a la zona reparada. Los resultados se expresan en $\%$ de cloruros respecto a peso de hormigón y \% de cloruros respecto a peso de cemento, suponiendo un contenido de 275 (p.c. 1) y $400 \mathrm{~kg}$ (p.c.2) de cemento por m3 de hormigón.

TABLA 5.

Resultados de cuantificación de cloruros en muestras extraídas del elemento B.

\begin{tabular}{ccccccc}
\hline $\begin{array}{c}\text { Distancia } \\
\text { (cm) }\end{array}$ & \%p.h. & \% p.c.1 & \% p.c.2 & \%p.h. & \%p.c.1 & \% p.c.2 \\
\hline 0 & 0,061 & 0,514 & 0,353 & 0,077 & 0,641 & 0,441 \\
1 & 0,062 & 0,519 & 0,357 & 0,091 & 0,762 & 0,524 \\
2 & 0,042 & 0,351 & 0,242 & 0,054 & 0,455 & 0,313 \\
$3^{*}$ & 0,086 & 0,716 & 0,492 & - & - & - \\
\hline
\end{tabular}

*hormigón en contacto directo con la armadura

La concentración de cloruros es muy similar en el punto 2 y en el punto 11. Ambos puntos siguen contaminados por cloruros con concentraciones en zonas próximas a la armadura ( $3 \mathrm{~cm}$ de recubrimiento) que llegan al límite del $0,6 \%$ de cloruros en peso de cemento considerado como valor límite si se supone un contenido de cemento de $275 \mathrm{Kg} / \mathrm{m}^{3}$ de hormigón (escenario más conservador). Esto no coincide con lo obtenido mediante técnicas electroquímicas donde no se detectaban procesos de corrosión.

Se observa, pues, que existen zonas del elemento B aún contaminadas por cloruros que, si bien no presentan síntomas de corrosión, si no son aisladas de la fuente de los cloruros o se limita la movilidad de estos, los cloruros podrán seguir acumulándose y comenzar a provocar daños por corrosión en las armaduras.

En este caso se pone de manifiesto la necesidad no solo de una inspección visual para la acotación de la zona de parcheo, sino ensayos que permitan cuantificar la fuente de contaminación, permitiendo a los agentes implicados la decisión de la selección de las zonas a reparar: i) zona deteriorada visualmente; ii) zona no deteriorada pero con el periodo de iniciación de la corrosión en marcha; iii) zona sin deterioro y sin corrosión, pero con cloruros suficientes para iniciar la corrosión en un futuro próximo.

\section{Elemento C}

En la tabla 6 se presentan los resultados de concentración de cloruros de las camas de asiento analizadas.

Se puede observar que la cantidad de cloruros acumulada en estos elementos es muy superior a la presente en las demás zonas analizadas. Es muy posible que los cloruros aportados como sales superficiales sobre la calzada percolen hasta depositarse en las camas de asiento de las vigas. La ausencia de una gran humedad puede favorecer que los clo- ruros no continúen difundiendo hasta hormigones de zonas inferiores. Estos altos contenidos en $\mathrm{Cl}$ - coinciden con que estos elementos eran los que presentaban un peor estado.

TABLA 6.

Cuantificación de cloruros en el hormigón de camas de asiento de vigas. Muestra obtenida con taladro para los dos primeros centímetros.

\begin{tabular}{lccc}
\hline Cloruros & ppm & \% p. h. & \% p. c. \\
\hline Pto. 1 & 1527 & 0,1527 & 1,28268 \\
Pto. 2 & 768 & 0,0768 & 0,64512 \\
Pto. 3 & 3449 & 0,3449 & 2,89716 \\
Pto. 4 & 1588 & 0,1588 & 1,33392 \\
\hline
\end{tabular}

En este caso, se pone de manifiesto que hasta que no existen altas cantidades de agentes contaminantes (en este caso cloruros) y por tanto deterioro, no se es capaz de detectar el problema mediante una inspección visual. Por otro lado, conocer estas cantidades de cloruros y su evolución en la estructura, permite no solo delimitar las zonas, sino averiguar la causa o fuente de llegada de estos cloruros, pudiendo trabajar también en la línea de evitarla.

4.

\section{CONCLUSIONES}

Se han presentado resultados de cuantificación de cloruros obtenidos en una obra afectada por corrosión en las armaduras del hormigón. Los datos han sido obtenidos in situ gracias a un equipo portátil de FRX.

Para contrastar los resultados se ha evaluado el estado de corrosión de las armaduras mediante un corrosímetro GECOR. Mediante este dispositivo se puede conocer el estado de los aceros antes y después de la reparación. Sin embargo, tanto la manipulación del corrosímetro como la interpretación de los resultados ha de hacerse por personal experto, como queda de manifiesto en el caso presentado, donde el dispositivo muestra que existe corrosión activa en zonas recientemente reparadas y ausencia de ella en las no reparadas todavía. La corrosión observada en las zonas reparadas se debe a que la repasivación de las armaduras saneadas aún no se había iniciado ( $24 \mathrm{~h}$ después de reparar) y que para comprobar la eficacia de estas reparaciones hay que medir parámetros de corrosión al cabo de tiempo suficiente para que dichas armaduras acaben este proceso de repasivación (las medidas realizadas 15 días después ya mostraban una disminución del 85\% de esas intensidades de corrosión).

El uso del equipo de pFRX es sencillo y rápido, lo que permite obtener in situ datos de concentración de cloruros y posibilita delimitar mejor la superficie a reparar por parcheo. Dado que en muchas ocasiones son los medios auxiliares los que suponen mayor coste en el global de las reparaciones, el equipo de pFRX supone un gran ahorro. Hay que recordar que los resultados dados por el equipo vienen expresados en ppm (partes por millón) con respecto a toda la muestra, mientras que los límites definidos por la normativa vienen expresados en porcentaje con respecto al cemento. Por ello, 
o se conoce perfectamente la dosificación del hormigón analizado o han de hacerse ciertas suposiciones que permitan obtener los resultados en las mismas unidades para compararlos con los valores límites establecidos en la EHE.

Conocer la cantidad de cloruros que existen en la estructura es fundamental a la hora de decidir las zonas a reparar. La reparación debe extenderse a todas las zonas en las que el contenido en cloruros en el hormigón en contacto con la armadura se acerque a los límites prescritos por la EHE. Más aún, conocer la cantidad de cloruros que permanecen en la estructura en las zonas donde no se proceda a la reparación por parcheo es básico para conocer cómo se desarrollará el deterioro en esas zonas en el futuro. Es posible que la cantidad de cloruros no sea la suficiente en ese momento para provocar la corrosión, pero alertan de si se debe ampliar o no la zona a reparar, evaluando de manera global la vida útil de la estructura mediante medidas cuantitativas y no bajo un examen visual que en ocasiones puede no ser objetivo.

En ese sentido, el equipo de fluorescencia de rayos $\mathrm{X}$ portátil permite monitorizar la concentración de cloruros en zonas deseadas con lo que podemos anticiparnos al daño por acción de estos contaminantes o a la despasivación de las armaduras.

\section{Agradecimientos}

El presente trabajo es fruto de las investigaciones realizadas en el marco del proyecto XRF-CODE (Aplicación de técnicas avanzadas de análisis de espectrometría por fluorescencia de rayos $\mathrm{X}$ a la reparación de estructuras de hormigón) financiado por el CDTI y desarrollado por la empresa RETINEO Ingeniería con la colaboración del Instituto Eduardo Torroja perteneciente al CSIC.5, 8, 15, 27, 32 Garamond 11

\section{Referencias}

[1] Sanchez, J., et al.(2017) Determination of reinforced concrete durability with on-site resistivity measurements. Materials and Structures, 2017. $50(41)$

[2] Angst, U., et al.(2009) Critical chloride content in reinforced concrete A review. Cement and Concrete Research, 2009. 39(12).

[3] Gonzalez, J.A., et al. (1996) Some questions on the corrosion of steel in concrete. Part II: Corrosion mechanism and monitoring, service life prediction and protection methods. Materials and Structures, 1996. 29(186).

[4] Pourbaix, M. (1990) Thermodynamics and corrosion. Corrosion Science, 1990. 30(10).

[5] Andrade, C., Sarria, J., \& Alonso, C. (1999) Relative humidity in the interior of concrete exposed to natural and artificial weathering. CCR, 1999. 29(8).

[6] Veluchamy, A., et al. (2017) Critical review on the passive film formation and breakdown on iron electrode and the models for the mechanisms underlying passivity. Journal of Electroanalytical Chemistry, 2017. 785.

[7] Pachón-Montaño, A., et al. (2018) Threshold concentration of chlorides in concrete for stainless steel reinforcement: Classic austenitic and new duplex stainless steel. Construction and Building Materials, 2018. 186.

[8] UNE 112010, Corrosión en armaduras. Determinación de cloruros en hormigones endurecidos y puestos en servicio. 2011.
[9] Chakraborty, S., et al. (2019) Use of portable X-ray fluorescence spectrometry for classifying soils from different land use land cover systems in India. Geoderma, 2019. 338.

[10] Wan, M., et al. (2019) Application of arc emission spectrometry and portable X-ray fluorescence spectrometry to rapid risk assessment of heavy metals in agricultural soils. Ecological Indicators, 2019. 101.

[11] Arenas-Islas, D., et al. (2019) Calibration of portable X-ray fluorescence equipment for the geochemical analysis of carbonate matrices. Sedimentary Geology, 2019. 391.

[12] Ravansari, R. \& Lemke, L.D. Portable X-ray fluorescence trace metal measurement in organic rich soils: pXRF response as a function of organic matter fraction. Geoderma, 2018. 319.

[13] Al-Eshaikh, M.A. \& Kadachi, A. (2011) Elemental analysis of steel products using X-ray fluorescence (XRF) technique. Journal of King Saud University - Engineering Sciences, 2011.23(2)

[14] Niziolek, L.C. (2018) Portable X-ray fluorescence analysis of ceramic covered boxes from the 12th/13th-century Java Sea Shipwreck: A preliminary investigation. Journal of Archaeological Science: Reports, 2018. 21.

[15] Čechák, T., et al. (2001) Analysis of fresco paintings by X-ray fluorescence method. RPC, 2001. 61(3).

[16] Havukainen, J., et al. (2019) Applicability of a field portable X-ray fluorescence for analyzing elemental concentration of waste samples. Waste Management, 2019.83.

[17] Block, C.N., et al. (2007) Use of handheld X-ray fluorescence spectrometry units for identification of arsenic in treated wood. Environmental Pollution, 2007. 148(2)

[18] Kalnicky, D.J. \& R. Singhvi (2001) Field portable XRF analysis of environmental samples. Journal of Hazardous Materials, 2001. 83(1).

[19] Suarez-Fernandez, G.P., et al. (2001) Analysis of major, minor and trace elements in coal by radioisotope X-ray fluorescence spectrometry. Fuel, 2001. 80(2).

[20] Yurdakul, E., Taylor, P.C. \& Ceylan, H. (2012) The Application of X-Ray Fluorescence to Assess Proportions of Fresh Concrete. in Civil, Construction and Environmental Engineering Conference Presentations and Proceedings. 6. 2012.

[21] Raupach, M. (2006) Patch repairs on reinforced concrete structures Model investigations on the required size and practical consequences. Cement and Concrete Composites, 2006. 28(8).

[22] Castro, P., et al. (2003) Macrocell Activity in Slightly Chloride-Contaminated Concrete Induced by Reinforcement Primers. CORROSION, 2003. 59(6)

[23] Qian, S., Zhang, J. \& Qu, D. (2006) Theoretical and experimental study of microcell and macrocell corrosion in patch repairs of concrete structures. CCC, 2006. 28(8).

[24] Schiebl, P., W.b. and Raupach, M. Durability of Local Repair Measures on Concrete Structures Damaged by Reinforcement Corrosion. ACI Symposium Publication. 145.

[25] Andrade, C., et al. (2012) On-site corrosion rate measurements: 3D simulation and representative values. Materials and Corrosion, 2012. 63(12).

[26] Andrade, C., et al. (2004) Test methods for on-site corrosion rate measurement of steel reinforcement in concrete by means of the polarization resistance method. Materials and Structures, 2004. 37(273).

[27] Feliu, S., Gonzalez, J.A. \& Andrade, C. (1996) Electrochemical methods for on-site determinations of corrosion rates of rebars, in Techniques to Assess the Corrosion Activity of Steel Reinforced Concrete Structures, N.S. Berke, et al., Editors. 1996.

[28] Andrade, C., et al. (1996) On-site measurement of the corrosion rate. Recent Advances in Bridge Engineering - Evaluation, Management and Repair, ed. J.R. Casas, F.W. Klaiber, and A.R. Mari. 1996.

[29] UNE 112083, Medición del potencial de corrosión libre en estructuras de hormigón armado. 2010.

[30] EHE-08, Instrución de Hormigón Estructural. 2008 